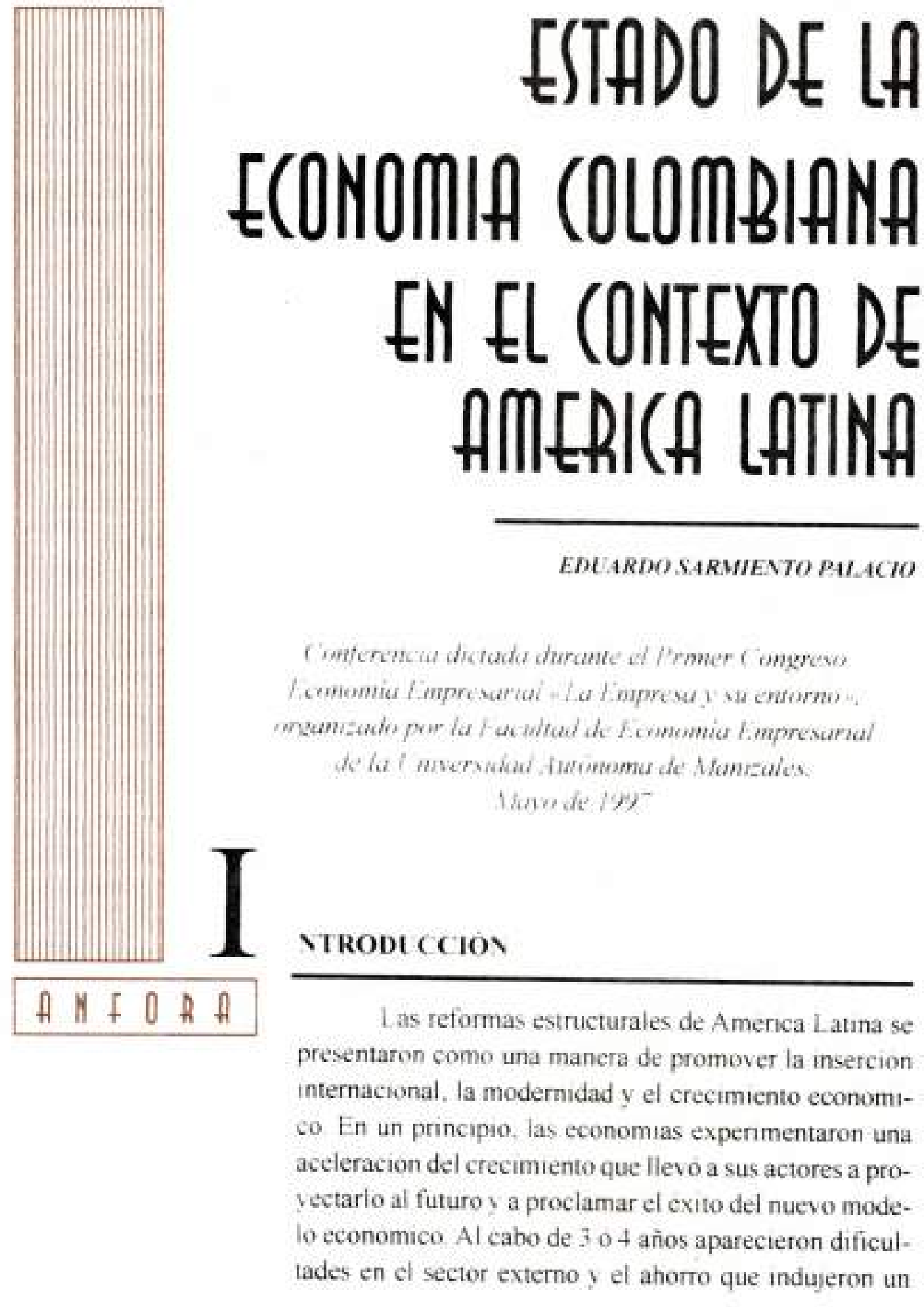


fuerte descenso de la actividad productIva que compenso con creces los aumentos iniciales. El balance no podia ser mas insatisfactorio. En los primeros seis años de la decada el producto de la region avanzo $3.0^{\circ} \mathrm{v}$ para fa parte restante se provectan tasas entre $35^{\circ} 0 \mathrm{y} 4^{\circ} \%$. De suerte que el crucumiento de la region durante la totalıdad de la decada sera cercano a $3.30 \%$. Esta cifra corresponde a un aumento de $1.2 \%$ del ingreso per capita que apenas compensa la caida de $1 \%$ registrada en la epoca perdida del 80 y muy inferior al promedio obtendo en las decadas del 50.60 y 70 . Ast como la decada del 80 fue la decada perdida del endeudamien10. la del 90 sera la mitad de la decada perdida del choque neoliberal

l.os mavores costos del modesto desempento de la region recaveron en los sectores laborales. En todos los paises se presentaron aumentos del desempleo y solo en unos pocos se registraron aumentos modestos def salario real El ingreso de los grupos laborales avanzo por debalo del crecimiento de la poblacion y la mayor parte de los beneficios del modesto erecimiento se concentro en el capital

En la actualidad la region pasa por un estado critico Las reformas estructurales no dieron los resultados buscados, o si se quiere, previstos. Por to demas, el mal desempeño de la economa y en parnicular del sector laboral. ha generado protestas y presioncs para modificar el modelo economico

La economia colombiana se mueve dentro de un contexto similar. La apertura comercial v cambiaria determino un cambio en la estructura productiva en contra de las actividades industriales i agricolas de alto valor agregado y desemboco en cuantioso deficit en cuenta corriente. En un principio el producto nacional se acelero. al cabo de tres años cayo y en la actuairdad pasa por un persistente proceso recesivo. Los mavores costos recaveron en el mercado laboral: en la agricuitura tomo la forma de una caida del saları reai y en el sector urbano en una expiosion del desempleo

Para bien o para mal, las reformas estructurales adoptadas durante la admunustracion Gavina, modificaron el funcionamiento de la economia colombiana. El marco actual es muy distunto al existente hace seis anos y para entenderlo es necesario examınar la evolucion reciente y anticipar los ajustes en lo que queda del siglo.

\section{CIMBIO ESTRLCTIRAL}

Colombia aparecio en el contexto latinoamencano como una de las economias mas reguladas y, al mismo tiempo. de mejor desempeño. Sus tasas de crecimiento, stn duda, las menos volatiles, solo fueron superadas por 
Brasil. Por lo demas, mostraba el historial de menores tasas de inflacion : de menos frecuentes crisis en el sectot externo

La estabilidad de la economia colombiana constitura una limitacion para retornar al modelo proteccionista Para que cambuar algo que habia funcionado adecuadamente? Tambien creaba, sin embargo, angustia en los organismos anternacionales, que no veran bien que la economia de mejor desempeño de Ametica Latina no entrara dentro del campo de las reformas Asi, Colombia fue el pais que mas se demoro en introduct las reformas. 1. a diferencia de la mavoria de las economias de la region. no lo hizo imputsado por las crisis sino por razones ideologicas
El desmonte arancelario en Co. lombia fue uno de las mas drasticos de America Latina El promedio de aranceles bajo en menos de un año de $38^{\circ} \circ$ a $122^{\circ}$. Adicionalmente. se modifico substancialmente la estructura relativa. Los aranceles reales de las materias primas y bienes intermedios bajaron en una mavor proporcion que los restantes Al final se llego a un arancel de $20^{\circ}$ 。 para los bienes finales, de $15^{\circ}$ o para los bienes intermedios y de $10 \%$ para las materias primas

La estructura arancelaria. en comjunto con la revaluacion del tipo real de cambio. propiciaron un desplazamento masivo de los sectores de alto valor agregado. Mientras en la industria se presento un debilitamiento en los productos intermedios, en la agricultu-

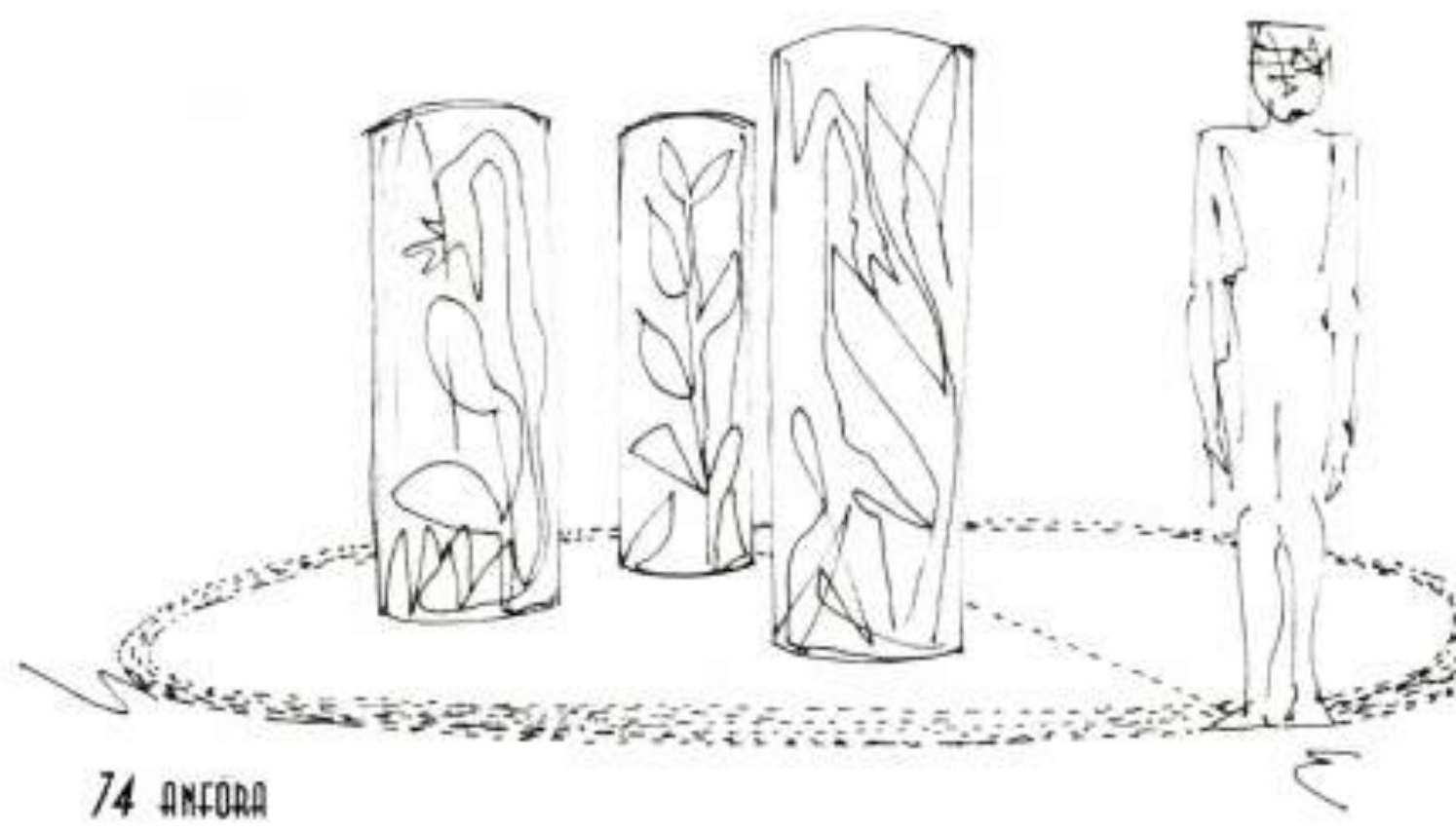




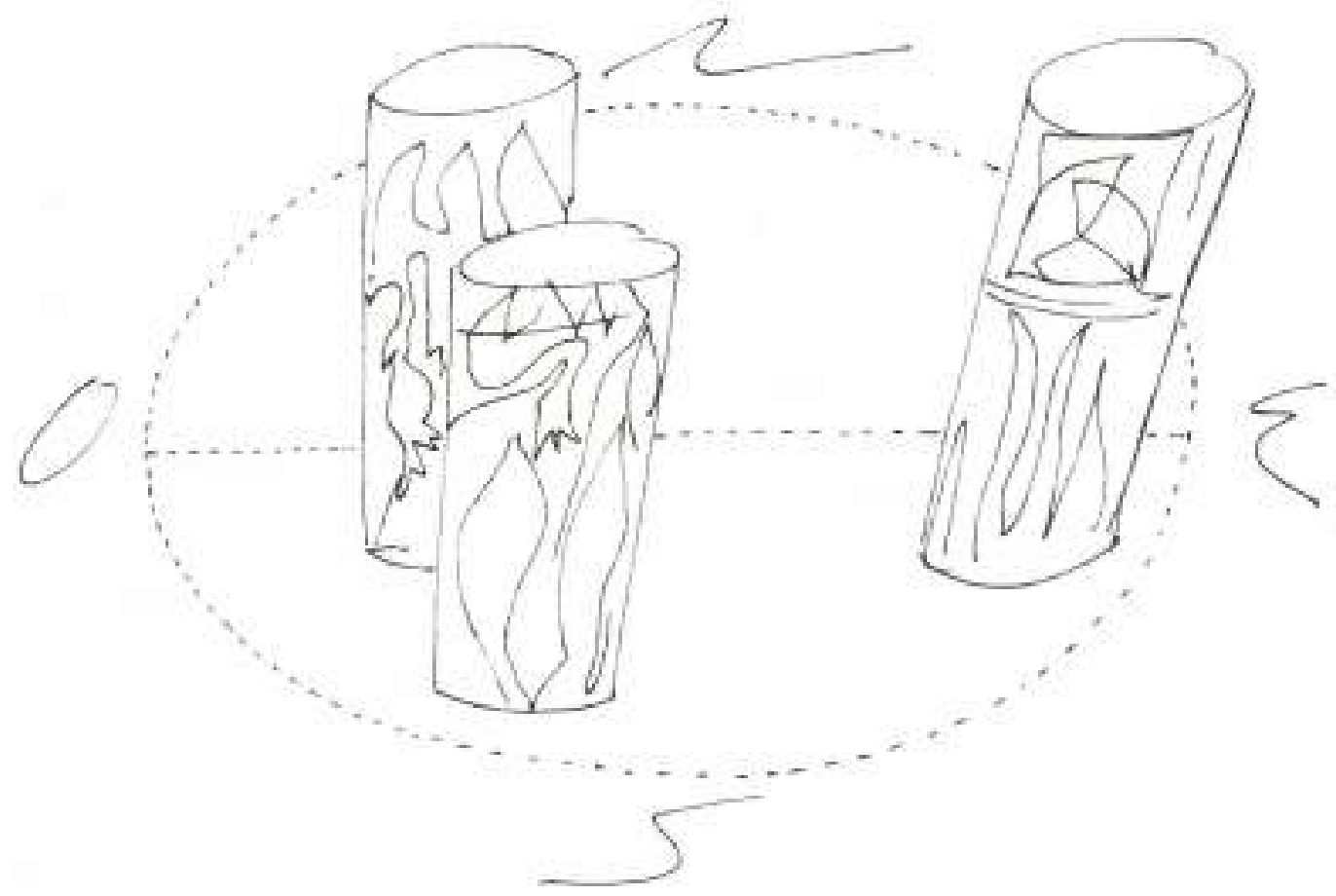

ra se regustro una caida sistematica del area de cuituvos transitorios. A cambio de ello se vieron favorecidas las actividades no transables y de ensamble La industria de matenales de construccion y los sectores de ensamble, como automoviles. sustancias quimicas y bienes de capital, avanzaron por encima del promedio Lo propro sucedio en la agricultura, en donde florecieron las actividades agromdustrales, que emplean los productos comerciales como insumos, al igual que los cultuvos permanentes que enfrentan dificulades de comercializacion extema como la yuca, el กิame y la papa

En fin, el experimento determtno un cambio fundamental en la composición productiva. Tanto en la indus. tra como en la agricultura se presento un desplazamiento masivo de las actividades de al to contenido de valor agregado hacia las actividades de ensamble y las actividades no transables.

La apertura se justifico como una forma de revertur el comportamiento de la productividad que en la decada de los 80 habua crecido por debajo del promedio historico. Se consideraba que la mayor disponibilidad y diversidad de los insumos y el aumento de la inversion inducidos por los menores precios internacionales traerian consigo un alza de la productividad que propiciaria la competitividad de los productos colombianos en el exterior. En efecto, la productividad (detinida como la relacion entre la produccion y el empleoj, tanto para la industra como para el conjunto de la economia, aumento signuficativa- 
mente con relacion al pasado. La productividad industrat. que en el pasado crecio $2^{0} 0$, en el penodo 1991-1995 lo hizo al $54^{\circ}$ \% y en los ultumos años por encima del $6 \%$ 。

No faltaron quenes prociamaran con base en esta informacion el exito y el milagro de la apertura El analisis detallado de la informacion revela algo mus distunto En general se encuentra que la elevacion de productividad tuvo lugar smultaneamente con el aumento de las importaciones y una caida del empleo. La explicacion es simple En mi libro uCrecumiento economico y aperturam (if ise muestra que el mayor crecimiento de la productividad se explica en su totalidad por la mavor utilizacion de insumos y productos intermedios importados. Si el calculo se realiza en terminos del salor agregado, el crecimiento de la productividad no cambia significativamente con relacion al pasado

Los resultados mas sorprendentes de la apertura se presentaron en la balanza de pagos. De acuerdo con las teoras de Heckscher-Ohlin y de Ricardo. que sirvieron de inspiracion a los promotores del experimento, la liberacion ocasionaria un cambio en favor de las actividades con ventaja comparatva. que supuestamente estan en capacidad de movilizarse en forma mas fac1l y en mayores volumenes. De esta manera. las exportaciones se amplianan

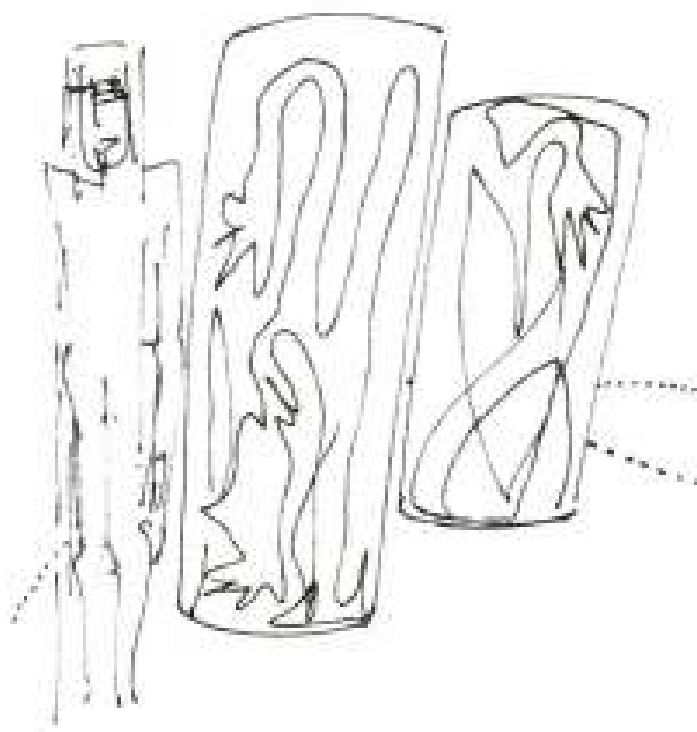

paralelamente al aumento de las importaciones

Las cosas ocurrieron en forma distinta Lias actividades productivas se trasladaron hacia los sectores de mavor tentaja comparativa que en el caso colombiano estan representados por los productos de menor complejidad tecnologica, que tienen menores posibilidades de demanda externa, como son los bienes no transables y las actividades de ensamble Pero, contrario a lo que se pretendia, las exportaciones quedaron relegadas a segundo plano. Al final surgio una estructura productiva altamente dependiente de las importaciones y que enfrentaba serias limitaciones para penetrar en los mercados externos. Tanto en la industria como en la agricultura, las importaciones se multiplicaron por 2 o $3 \mathrm{y}$ las exportaciones se estancaron. Se configuro un 
racterizan por una gran demanda externa. El cambio en la estructura productiva trajo consigo un aumento de las exportaciones muy superior al de la produccion. El producto nacional se acelero gracias a un crecimiento mavor de las importaciones y estas se lograron sostener por el clevado creclmiento de las exponaciones. De esta forma, la economa ha logrado mantener elevadas tasas de crecimiento con mayores tasas de crecimiento de las importaciones y con un equilibrio en la balarza de pagos

Volvamos a la economia colombiana Frente a la tendencia antes descrita y la eminencia al colapso, las autoridades monetarias adoptaron un severo programa de restriccion monetaria. El Gobierno pretendta reducir el ingreso y la demanda agregada para ampliar las exportaciones y reducit las importaciones. Este manejo se reflejo

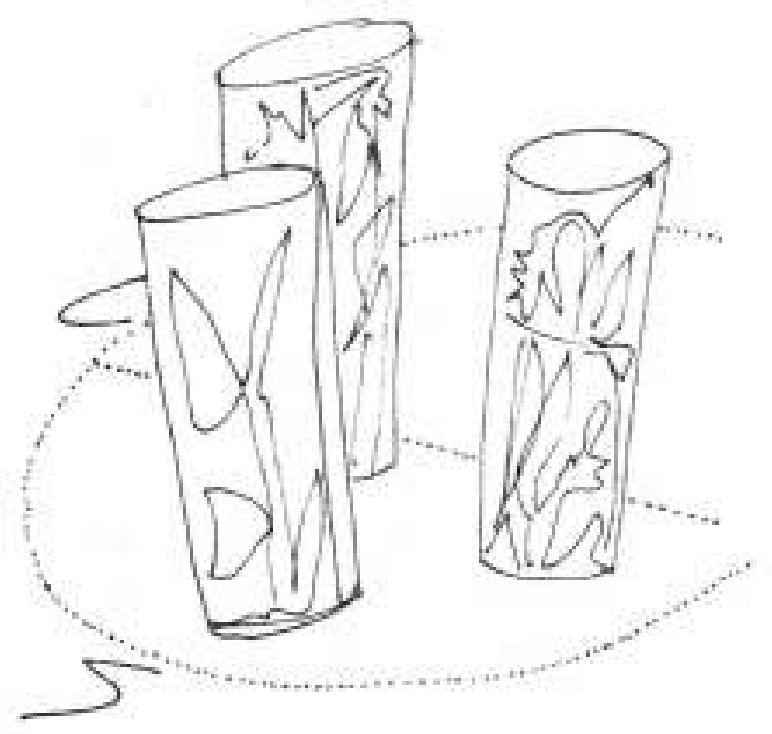

en un aumento de los medios de pago de menos de $10 \%$ y en tasas de interes activas de $50^{\circ}$. En un plazo relativamente corto se observo el debilitamiento de la construccion y de las ventas del comercio que revelaban un claro decalmiento de la demanda Luego el proceso se traslado a la industria. los servicies y las importaciones

Sin embargo, los efectos sobre el secuor externo no correspondian a los descos, o si se quiere, a los propositos. I. as altas tasas de interes indujeron una entrada de capitales que indujo la tes aluacion del tipo real de cambio que. a su turno, creo mas estimulos para traer capitales La economia entro en un proceso indefinido de endeudamiento y revaluacion. A los efectos de la apertura, se agregaron las altas tasas de interes y la revaluacion. y los tres elementos en conjunto precipitaron la economia en una recesion continuada sin precedentes

Lo curioso es que esta politica no logro corregir significativamente el deficit en cuenta corriente Al desmonte arancelario y a la revaluación que venia de atras se le adiciono mas revaluacion. La reduccion de las importaciones y la mejoria de la balanza de pago provinieron de la caida de la actividad productivay no del ajuste de los precios relativos. Por eso, a finales de 1996 la stuacion cambiaria continuaba siendo critica. En un momento de 
profunda recesion el deficit en cuenta corriente llegaba a $5.5^{\circ}$ o del PIB A menos de que se corrija la revaluacion. tan pronto como la economia experimente algun upo de reactivacion, ef deficit en cuenta corriente volvera a surgir y en ese momento se darta el colapso

\section{EVOLICIÓN DE LAACTIVIDAD PRODICTINAEN LOSILTIMOS DOSAÑOS}

Las secuelas estructurales de la apertura empezaron a retlejarse a principios de 1995. En los primeros meses se observaron la carda de los precios de la bolsa. la reduccion de las zentas de viviendas y el debilitamiento de las ventas del comercio. Mas tarde. se extendio a la industria que recoge a todas las actividades de la economia y al empleo urbano Luego, el proceso adquirio una dinamica acelerada Al final del aǹo era evidente que la economia habia entrado en plena recesion

En 1996 el deterioro de la economia adquirio visos alarmantes. La produccion industriat descendio sistematicamente y en los diez primeros meses revelaba una caida de 250 aun mas dicjente, en septiembre y octubre se registraron indice negativos de $7^{\circ}$ o y todos los sectores a dos digitos descendian. La construccion revelaba ca!das de $35 \%$ sobre caidas similares en el año antenor $\mathrm{y}$ las de comercio $35^{\prime \prime}$ " A tinal del año la crisss se extendia a todos los sectores sint excepcion El desempleo urbano aumento $59^{\circ} \circ \mathrm{y}$ el empleo descendio $1 \%$ El consumo de energla en las cuatro grandes ciudades bajo $4^{\circ} 0$

tn la agricultura los episodios cvolucionaron un tanto distinto. El sector no ha logrado asımilar el golpe de la apertura En 1996, por cuarto año consecutivo, descendio el area de cultivos transitorios.

La caracteristica sobresaliente en la recesion no esta tanto en la maynitud como en la duracion. la persistencia y el agravamento. Sin duda, se trata de un proceso que no genera correctivos. En in estado recesivo lo normal es que el tipo de cambio se devalue. la intlacion descienda y la tasa de interes baje. Nada de esto ha ocurndo en las magnitudes necesanas por-

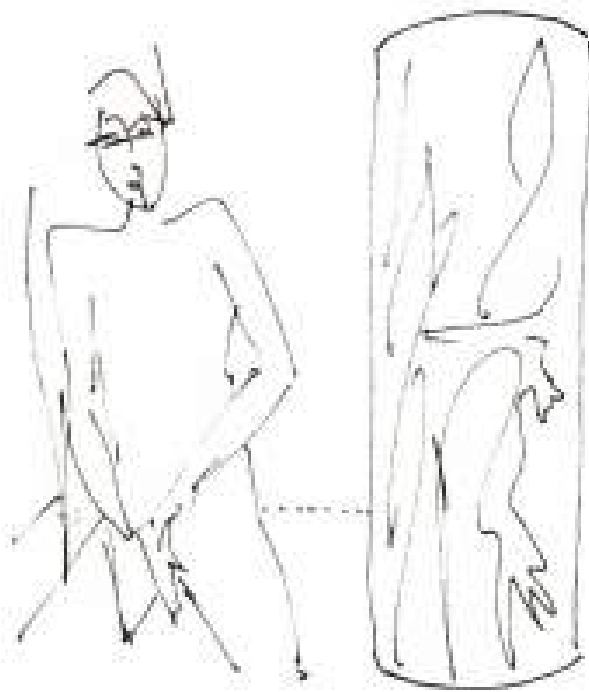


que las politicas y las retormas neoliberales no han logrado ajustarse a la realidad de la economia colombrana. Los mos imientos de ta tasa nomtnal de cambio no arrastran la intlacion que es determınada por tactores inerciales. $y$ los movimiento de capita. les no nivelan las tasas de interes inter. nas debido a los poderes monopolicos que impiden el descenso en las tasas de inieres, en especial las activas De hecho, se ha perpetuado un estado estructural de altas tasas de interes y revaluacion que tienden a mantener la recesion e incluso a agravarla

\section{INFLACION}

E) Tesultiado mas sobresaliente de las reformas estructurales de America Latina fue el descenso de la inflacion En la mayoria de los palses se aprecia una caida notoria de los indices de aumento de precios: Por lo demas. las hiperinflaciones de Bolivia, Arigentina. Peru y Brasil se convirtieron en alzas de menos de un digito.

A Colombia no le fue bien en esta danza Durante dos decadas la inflacion colombiana aparecio como una de las mas bajas y estables de la region
Ahora. corresponde a una de las mas altas y mas estables Al parecer, los avances institucionales realizados para estabilizar la inflacion airededor de la rendencia histonca impidieron que ella se disparara. pero tambien se convirtieron en restriceion para su descenso No obstante que la proondad de la politica economica ha girado en la reduccion de la intlacion, los resultados han sido exiguos En los ultumos cuatro años la inflacion apenas bajo 2 puntos porcentuales con relacion al promedio historico de las ultumas dos decadas.

Curiosamente. éste desempeño sucedio en un periodo en que entro en vigencia la norma constitucional que establece la autonoma de la banca central y le asigna como priondad unica bajar la intlacion Nunca antes se habian aplicado los poderes monetanos y cambiarios con tanta discrecionalidad e intensidad para lograr un proposito inflacionano. En unas epocas se adoptaron severas restricciones monetarias que se manifestaron en altas tasas de interes $y$ precipitaron la economia en recesion. En otros periodos, se congelo practicamente el tipo nominal de cambio. La primera terapia

\section{AKEOAPA}


se reflejo en severas recesiones y la segunda en cuantiosas revaluaciones ? ninguna de ellas consiguio el cumplimiento de las metas intlacionaras ofisiales, las cuales se superaron sistematucamente durante cinco años Hoy en dia es evidente que los esfuerzos y los costos monetaros no guardan relacion con los resultados. Apenas se logro reducir la inflacion en dos puntos porcentuales con respecto al promedio hisiorico de las uitumas dos decadas

Los desaciertos se originan en que los economistas colombianos no han entendido la naturaleza de la inflacion en Colombia No se ha logrado superar la vision de los libros de texto segun la cual la inflacion es un problema de escaseces $y$, como tal,

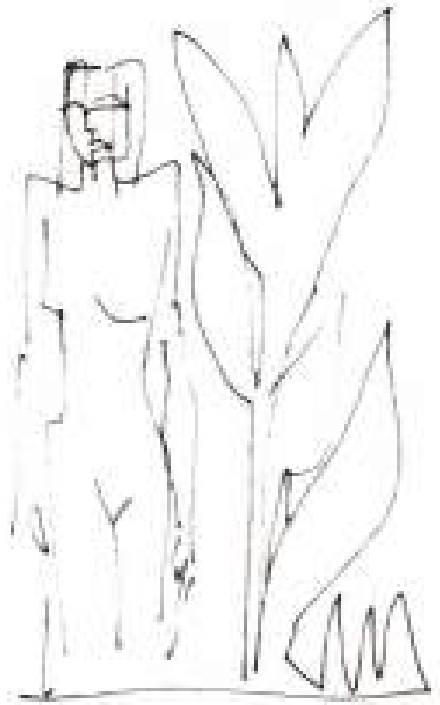
se corrige con politicas contraccionistas de demanda, bien sean monetarias, fiscales o cambiarias En cierta forma se ignora toda la esidencia empirica que muestra que se trata de un fenomeno inercial, que como lo dice su propio nombre trende a sostenerse por si misma En tales condiciones, la aplicacion de politicas restrictivas se manifiestan en recesion. las cambiarias en revaluacion y ninguna de ellas consi- gue reducir significativamente la inflacion

B origen de la inflacion inercial es el largo periodo de ajuste de los salanos y otros precios administrados Fstos se ajustan de acuerdo con la inflacion del pasado y luego determinan la del futuro. Asi, la dinamica del proceso esta dada por el periodo de ajuste de los salarios

Fl proceso puede desactivarse entonces reduciendo los periodos de ajuste de los salarios Tanto la magnitud del ajuste como las frecuencias serian menores Luego la, menor magnitud de los ajustes bajarian la inflacion y luego esta se transmitiria a los periodos siguientes graclas al acortamiento de los periodos de ajuste. Al final, se configuraria un proceso declinante de la inflacion que podria colocarse facilmente a menos de la mitad en un año

La inflacion es el producto de errores en el manejo de la politica economica y en las expectativas de los individuos que no podian persistir indefinidamente En América Latina, que se caracterizo durante mucho tiempo como el campeón de la inflación, la dolencia se ha venido extungutendo y 
cada vez tiene menor importancia real. En la actualidad el promedio de la inflacion de ia regton es de $14^{\circ} \mathrm{o} \mathrm{y}$ en el corto plazo llegara a menos de $10^{\circ}$. La mayona de los passes estan operando con los niveles inferiores a un digito En Colombia mo se ha conseguido lo mismo porque se ha persistido en un diagnostico equis on ado de la dolencia. Si finalmente se entendiera el origen inercial de la intlacion s se actuara en consecuencia, tai como lo mostramos a lo largo de la seccion. Ia inflacion podria colocarse fautmente en menos de $10^{\circ} \%$ en un pliza de un ano

\section{POLITICA FISE IL}

Una de las anosactones mas importantes de fa nueva Constitucion esta en la descentralizacion admumstratwa y en el fortalesimiento del gasto social. En las normas se establece claramente que los ingresos tributarios deberan destunarse en porcentales crecientes a los municipios y a los gastos en salud y educacion. Otra cosa es que la iniciativa no sea v able dentro de una estructura tributara de corte neoliberal

Las dos admunistraciones antenores propiciaron un pertil tributano tipo Reagan. La reforma de 1986, adoptada cuando Gavina se desempeñaba como Ministro de Hacienda, al igual que en las posteriores revisiones, no se ahorraron esfuerzos para debilitar ef impuesto a la renta Se desmonto la progresividad tributaria. se elimino el impuesto al patrimonio y se eximio a una gran parte contribuyente de presentar deciaracion que es el medio mas efectivo de control de elevacion. Por lo demas, la apertura significo el desmonte def impuesto a las importaciones y la reduccion de los aranceles, que en el pasado constituveron las principales fuentes de ingresos oficiales

Estas condiciones afectaron senamente la discrecionalidad tributara. La dinamica del gasto propiciado por la Constitucion no ha tenido una contrapartida en el aumento de los recaudos. La reforma tributaria adoptada por la admunistracion Gaviria. que estipulo una sobretasa del impuesto a la renta y la elesacion del IVA, no logto modificar la inclasticidad de los recaudos Luego. la reforma adoptada durante la administracion Samper. cuando Perry se desempeñaba como Ministro de Hacienda. contemplo una elevacion adicional del IVA en dos puntos y no consiguio siquiera aumentar el recaudo con relacion al año anterior. Asi, en el periodo 1990 - 1996 los ingresos tributarios en terminos del PIB solo aumentaron 1.5 puntos porcentuales, mientras que el gasto lo hizo en 4 puntos percentuales i Como consecuencia, en 1996 aparecio un deficit del Gobierno central de $4.5 \%$ del PIB y otro del sector publico consolidado de $2.5 \%$

\section{AKFOAA}




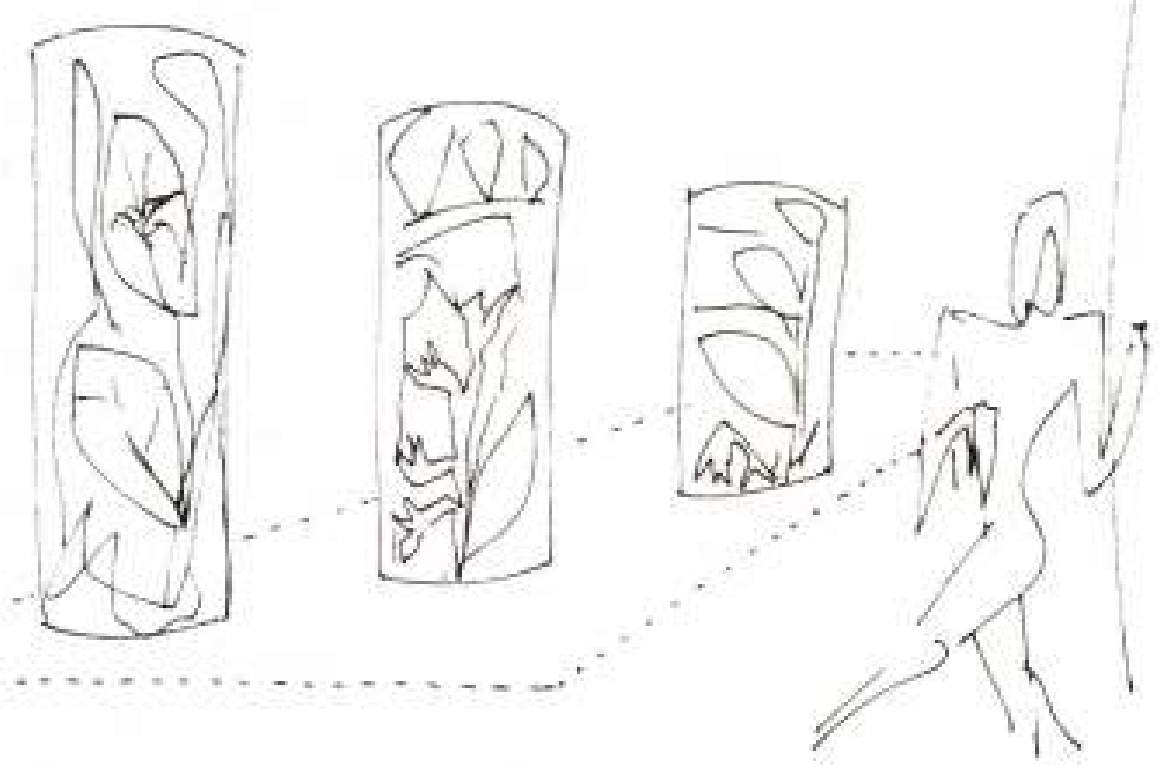

del PIB. Estas condiciones llevaron a decretar una emergencia economica para introducit nuevos ajustes tributarios y reducir et deficit fiscal. Los estumativos iniciales indican que esta vez tampoco va a ser La emergencia no lograra incrementar los recaudos en mas de $0.5^{\circ} \circ$ de PIB y no conseguira reducir et deficit fiscal en mas de $1 \%$ del PIB. En su lugar. contribura a prolongar y agravar ta recesion

La solucion al problema fiscal no es algo que pueda lograrse en pocos dias. En la practica se plantea una gran reforma tendiente a desmontar el mandato constitucional que obliga a destinar porcentajes crecientes de los ingresos tributarios al gasto social y a los municipios. De otra manera, el recorte del gasto publico significaria una caida monumental del gasto en la infraes- tructura. y en particular de la infraestructura val por ser la mas tacil de recortar. Ast mismo, se requeriria una gran retorma fiscal tendiente a elevar la elasticidad de los tributos Nada de esto puede realizarlo un solo gobierno En cualquier caso se requiere un cambio fundamental en el consenso que lleto a ampliar el gasto social y a concentrar los recaudos en el impuesto indirecto

\section{LA EXPERIENCIA DE AMÉRICA I.ATIVA}

La apertura fue una experiencia multiple y en cierta manera simultanea. Todos los paises de America Latina procedieron a reducir los aranceles y liberar el mercado cambiano. Por lo demas, los resultados tuvieron lugar 
dentro de fisonomias y comportamientos similares. Las estructuras productivas se movieron en favor de las actividades no transables y de alto componente importado, o si se quiere, de baja complejidad tecnologica Por eso. el denominador comun es el crecimiento en la industria y la agricultura por debajo del promedio de la cconomia Adicionalmente, aparecen cuantiosos deficit en cuenta corriente y caidas notables en la tasa de ahorro

Estos daños estructurales se ocultaron y, en cierta forma, sus manifestaciones se postergaron mediante la financiacion del deficit en cuenta comiente con entradas de capital. Las economias quedaron expuestas a un exceso de gasto sobre el producto interno bruto que ocasiono una fuerte expansion de las actividades no transables y de las actividades de ensamble. lo que

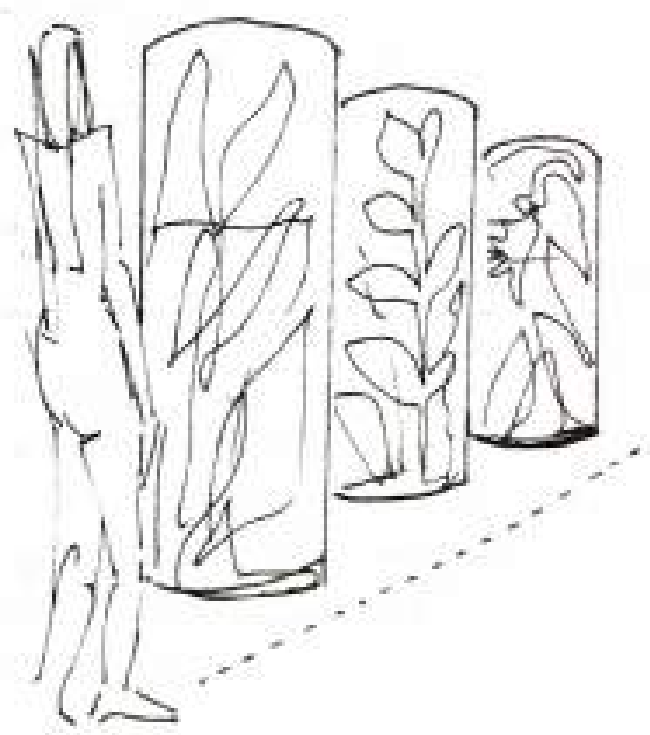

compenso el bajo desemperio de la industria y la agncuitura de aito valor agregado, incluso permitiendo sostener tasas de crecimiento superiores a las del pasado. Este resuitado se consiguio sin embargo, a cambio de un comportamiento explosivo de la balanza de pagos, mas concretamente. configurando un circulo vicioso en que la revaluacion conduce a un mayor deficit comercial $y$ este a una mayor revaluacion

Mexico que aparecio en los reportes de los organismos internacionales y en las revistas especializadas como "The Economist" como el modelo de apertura ideal, fue el encargado de mostrar que, al igual que en la decada de los 80 , este proceso no era sostenible. En el cuadro I se observa que esta economra se vio expuesta a un deficit en cuenta corriente creciente que logro financiarse sin problemas hasta 1993. Sin embargo, en 1994 las entradas de capitales fucron muy inferiores al deficit. dando lugar a la caida de las reservas internacionales que creo serias dudas sobre la capacidad del Gobierno para mantener el desequilibrio cambiario. Como consecuencia se generaron expectativas de devaluacion que llevaron a sacar los fondos del pais y a reducir los ingresos. Finalmente, las autoridades economicas no tuvieron mas alternativa que soltar la banda cambiaria y hacer efectiva la devaluacion en cası $100^{\circ} \%$.

\section{AMFOAA}




\section{Cuadro \# 1}

\section{BALANZA EN CUENTA CORRIENTE Y MOVIMIENTO DE CAPITAL (Millones US\$)}

\begin{tabular}{|lrrrrrrr|rrrrrr|r|}
\hline ANNOS & \multicolumn{9}{|c|}{ BALANZA CUENTA CORRIENTE } & \multicolumn{5}{c|}{ ENTRADA DE CAPITAL } \\
\hline & 1990 & 1991 & 1992 & 1993 & 1994 & 1995 & 1996 & 1990 & 1991 & 1992 & 1993 & 1994 & 1995 & 1996 \\
ARGEN & 1903 & -672 & -6677 & -7479 & -10500 & -3300 & -4200 & 1476 & 3301 & 12213 & 10047 & 10500 & -400 & 5840 \\
MEXICO & -8413 & -13890 & 24919 & -23489 & -28500 & -215 & -1000 & 11643 & 21882 & 26664 & 29531 & 19500 & -16900 & 1700 \\
VENEZ & 8305 & 1769 & -3747 & -1785 & 4090 & 1725 & -1680 & -5361 & 4562 & 717 & 1800 & -4505 & -3660 & 7255 \\
COL & 557 & 2363 & 925 & -2234 & -3020 & -4200 & -4660 & 53 & -527 & 167 & 2213 & 3075 & 4565 & 4910 \\
PERU & -1339 & -1900 & -2363 & -2217 & -2540 & -4238 & -3470 & 1624 & 2837 & 3081 & 2715 & 4041 & 3776 & 5110 \\
CHILE & -744 & -157 & -940 & -2416 & -639 & 160 & -2395 & 3075 & 1404 & 3487 & 2838 & 3855 & 921 & 3700 \\
BRASIL & -3809 & -1443 & 6266 & -637 & -1153 & -18136 & -20300 & 5054 & 1640 & 8802 & 9041 & 7696 & 31109 & 26300 \\
CHILE & -744 & -157 & -940 & -2416 & -639 & 160 & -2395 & 3075 & 1404 & 3487 & 2838 & 3855 & 921 & 3700 \\
BRASIL & -3809 & -1443 & 6266 & -637 & -1153 & -18136 & -20300 & 5054 & 1640 & 8802 & 9041 & 7696 & 31109 & 26300 \\
\hline
\end{tabular}

\begin{tabular}{|lrrrrrrr|}
\hline AÑOS & \multicolumn{1}{c}{ VARIACIONES RESERVAS INTERNACIONALES } \\
\hline & 1990 & 1991 & $\mathbf{1 9 9 2}$ & $\mathbf{1 9 9 3}$ & 1994 & $\mathbf{1 9 9 5}$ & 1996 \\
ARGEN & 21476 & 2629 & 4549 & 2568 & 0 & -3700 & 1650 \\
MEXICO & 3213 & 7992 & 1745 & 6042 & -9000 & -17115 & 700 \\
VENEZ & 2944 & 2225 & -1030 & 15 & -415 & -1935 & 6045 \\
COL & 610 & 1836 & 1092 & -21 & 55 & 365 & 250 \\
PERU & 285 & 937 & 718 & 498 & 1504 & -462 & 1640 \\
CHILE & 2331 & 1247 & 2547 & 422 & 3216 & 1081 & 1305 \\
BRASIL & 1245 & 197 & 15068 & 8404 & 6243 & 12973 & 6000 \\
CHILE & 2331 & 1247 & 2547 & 422 & 3216 & 1081 & 1305 \\
BRASIL & 1245 & 197 & 15068 & 8404 & 6243 & 12973 & 6000 \\
\hline
\end{tabular}


El orro caso es Argentina ton el cuadro I se observa que el defict en cuenta corriente se financio comodamente hasta 1994, cuando su monto correspondia practicamente a los ingresos de capitales En 1995 las entradas de capitales se veron reducidas por el colapso mexicano y la economia se vio expuesta a una caida vertical de las reservas internacionales. La contraccion monetaria resulto en un alza de las tasas de interes que precipito la economia en recesion. En $1995 \mathrm{cl}$ producto interno bruto descendio $50^{\circ}$ y tuvo su manifestacion mas dramatica en el desempleo que llego a $195^{\circ} \mathrm{o}$

En Mexico la correccion del desequilibrio se hizo por el camino de la devaluación y en Argentina por la contraccion monetaria y las altas tasas de interes. En ambos casos se demostró que el mantenimiento de un cieno equilibrio cambiario solo es posible con tasas de crecimiento infenores a los promedios historicos.

\section{Algo similar} ocurno en Peru. Laeconomia peruana experimento una fuerte reactivacion en el periodo 1993 - 1995, cuando registro tasas de crecimiento de $5.8 \%$. $13.9 \%$ y $7.7 \%$. Al mis- mo tiempo. se presento un aumento persistente del deficil en cuenta corriente que llego a US\$4 237 millones en $199518 \%$ del PIB | Las autoridades economicas no tuvieron mas alternativa que detener el proceso de recuperacion para moderar la tendencia del deficit En 1996 la economia solo crecio $2 \%$, 10 que significo el final de la bonanca.

El proceso brasilero ha sido mucho mas lento. En los seis primeros años de la decada la economia crecio a un modesto $2.7^{\circ}$. Aun asi. la apertura comercial y la revaluacion han traido consigo un persistente aumento del deficit en cuenta corriente que en la actualidad llega a US\$20,000 millones (2 $5^{\circ}$ u del P(B). De donde se deduce que si la economa pasara a tasas de crecimiento cercanas a $5^{\circ} \%$, el deficit se doblaria colocando la economa en la zona de candela. Sin duda, el sector exferno aparece como una seria restriction para que en otra hora la mas dinamica economia de America Latina recupere las tasas de crecimiento del pasado

La excepcion de la regla es Chile que ha logrado mantener altas tasas de creci-

\section{Q6 AMFOAD}


miento en las exportaciones y las importaciones. La explicacion esta en la enorme ventaja comparativa en productos de clima de estaciones que tienen una alta demanda externa Gracias a ello la economia logro mantener elevadas tasas decrecimiento en las exportactones sin absorber grandes recursos de los sectores. El crecimiento del producto ha coexistido con tasas de creclmiento muy superiores de las importaciones $y$ las exportaciones.

La gran pregunta es hasta donde puede mantenerse el modelo chileno Mientras las exportaciones crezcan a tasas superiores al producto nacional Este comportamiento ha sido posibic gracias a la enorme ventaja comparatıva de las exportaciones chilenas en productos de alta demanda, que estan expuestos a rendimientos decrecientes Cada vez sera mas dificil sostener ratmos de crecimiento de las exportaciones que dupinquen los del producto Algo de esto se ha venido observando recientemente, Las tasas de crecimiento del producto en los ultumos cuatro años siguen siendo superiores a $5 \%$ pero infenores al promedio de los ultimos 10 años y para 1997 se puede esperar una de- clinacion mayor Inevitablemente, la economia chilena tendera a estabilizarse en tasas de crecimiento cercanas e incluso inferiores a $5^{\circ}$.

\section{MERCADO LABORAL EN LA REGIÓN}

El resultado mas perverso de la apertura se dio en el mercado laboral. De acuerdo con la teoria de las ventajas comparativas. la liberacion comercial deberia promover un cambio en la estructura productiva en favor de las actrvidades con mavor ventaja comparatıva que tienen mayores niveles de productividad. De hecho, la fuerza de trabajo pasaria a obtener mayores remuneracionès Sin embargo, en un mundo expuesto a limitaciones en la demanda el resultado es muy distinto. L.as posibilidades de exportacion se concentran en los productos de cierta complejidad tecnologica y los paises en desarrollo solo estan en condiciones de competir en esas areas con menores salanos

Los aranceles en la practica no son mas que un medio para morigerar la caida del salario real Por eso mismo. el desmonte 
tanifario crea grandes presiones en contra del salario real En unos casos se manifiesta en reducciones efectivas de! salano real y en otros en desempleo En cualquiera de los dos casos determina el deterioro de los ingresos del trabajo con respecto al producto nacional

Lo anterior tienc una clara confirmacion en las cifras mas recientes de la region. En general se encuentra que el salario real ponderado por las pobiaciones de los paises se mantiene relativamente constante En todos los casos. con excepcion de Chile, el salane real crecio por debajo del $1 \%$ Al mismo tiempo, se observa que el desempieo aumento, en Argentina se tripico. en México se duplico y en Colombia se elevo en $50 \%$. En el comjunto de fa region la tasa de desempleo llego al nivel mas alto en las ultumas dos decadas, incluso superior a la registrada en las epocas de la crisis de la deuda externa.

En fin. estamos ante un modelo economico que se manifiesta en el estancamiento del salario real y en la profundizacion del desempleo. El aumento de los ingresos del trabajo resulto inferior al crecimiento de la poblacion y el modesto crecimiento del ingreso per capita del $1 \%$ se concentro totalmente en el capital

No menos diciente es la calidad de la mano de obra. En todas partes la apertura destruyo el empleo industrial. dejando la economa sin fuentes permanentes de ocupacion Las posibili. dades de empleo se reducen a las actlvidades informales y a las bonanzas pasajeras de la construccion. No solamente se ha aumentado el desempleo s se han estancado las remuneraciones. sino que se ha afectado negauvamente la permanencia y la estabilidad.

Muchas de las dificultades politicas de Amenca Latuna se explican por el deterioro del mercado de trabajo. Estamos ante un modelo economico cuvo equilibrio esta condicionado a reducciones significativas del salario real I. a alternativa es la recestion y el desempleo A su turno, las politicas recesivas de los góbiernos para bajar el salario real han generado serias manifestaciones de descontento que no son de facil manejo en el sistema democratico

\section{ESTADOACTIAL}

No es dificil sintetizar el estado de la region. Al cabo de 5 años es evidente que el modelo de apertura no ha dado los resultados previstos. En el unico campo en que se ha avanzado es en el de la inflacion que ha dejado de ser problema La inflacion promedio de America Latina es menos del $14 \% \mathrm{v}$ las hiperintlaciones dejaron de existir. El drama esta en que el modélo dejo a las

\section{Q8 ANFGDA}


economias creciendo a mitad de las tasas del pasado, debilito la industria y la agricultura y ocasiono un grave detenoro en el mercado laboral. Hoy en dia las economias no disponen del dinamismo de las decadas del 60 y el $70 \mathrm{y}$ es. tan expuestas a serios conflictos por el deterioro creciente de los grupos laborales.

El tipo de crecimiento que surgio de la apertura fundamentado en la alta sustitucion dei valor agregado nacional no tiene mucha solidez El dinamismo resulta de la especializacion de la economia en los procesos mas faciles, como sena el ensamble Ası. la expansion de la produccion se ortigina en la ampliacion de las importaciones de productos intermedios que vendrian a reemplazar la parte mas elaborada de la produccion y eliminar cuelios de botella. Las economas quedaran en posicion de acelerar el crecimiento economico mediante la mavor disponibilidad de importaciones La contradiccion esta en que las aperturas propician un cambio en la composicion productiva que desestimula las exportaciones. De hecho. la aceleracion del crecimiento se manifiesta por lo general en un deficit en cuenta corriente insostenible. Hasta cl momento la excepcion ha sido Chile porque ha tenido condiciones economicas : politicas especiales que tho pueden exienderse al resto de paises de America Latina

Los resultados del modelo han modificado la acritud de sus promotores. Hace un tiempo se justificaba como un medio para promover el progreso y la modernidad Hov en dia, en cambio. se justifica porque no hav otra alternativa ante las nuevas realıdades de la globaindad. Tan ziento es esto que la

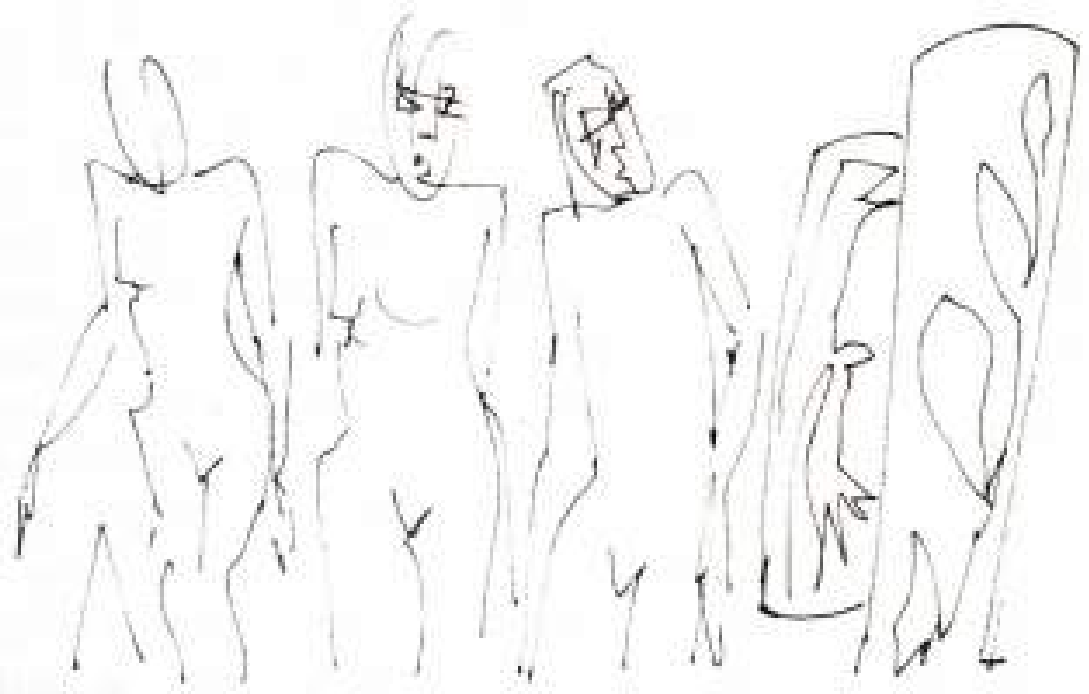




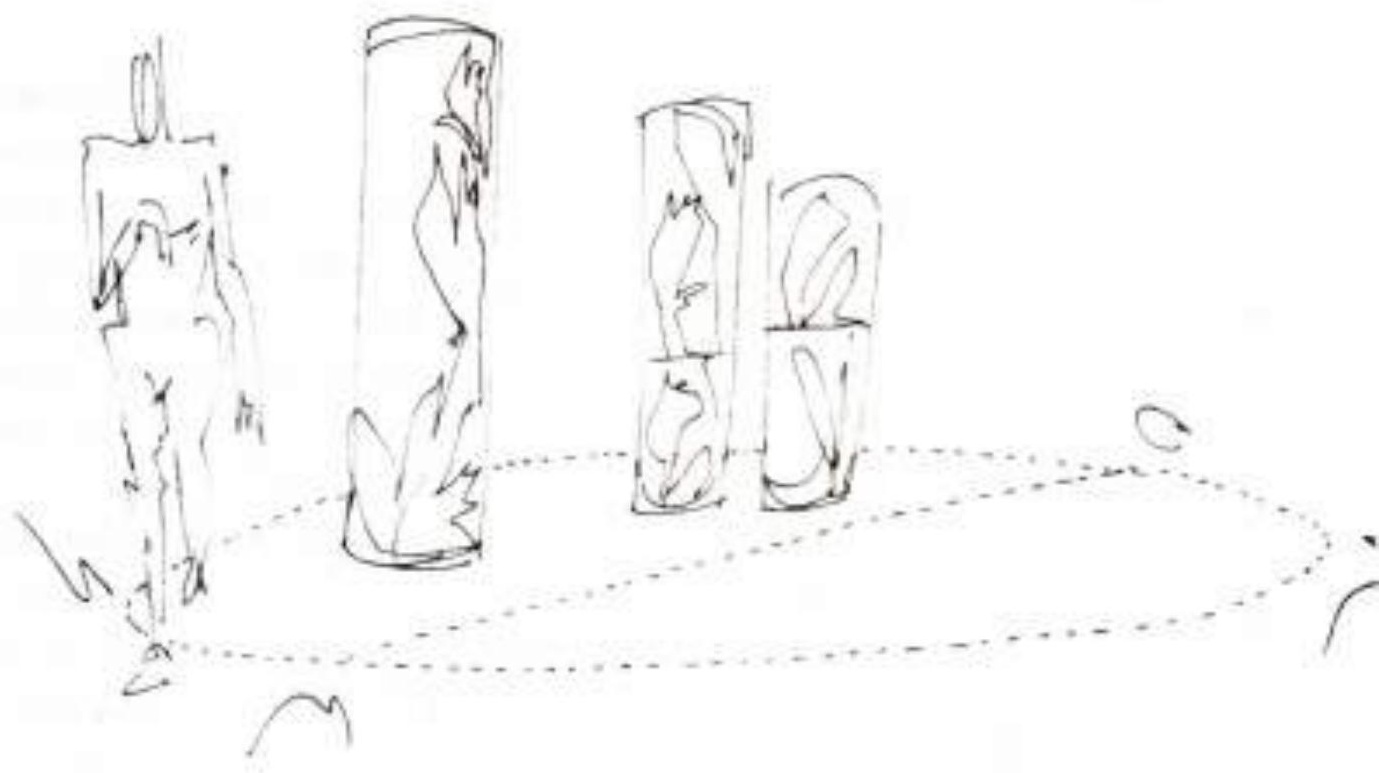

recomendacion de los organismos intemacionales ante los resultados neigativos del modelo es resistif hasta las ultimas consecuencias, independientemente de los resultados, Por otra parte. el bajo dinamismo del modelo v sus sesgos contra los ingresos laborales ha creado un gran descontento popular. En cierta forma. los paises se enfrentan ante la contradiccion de un modelo impuesto desde afuera que afecta negativamente a las grandes mavorias

Ciertamente, el modelo protecclonista extremo no es viable dentro de los avances en materia de transporte : comunicaciones. Las empresas estan en capacidad de ensamblar el producto en cualquier lugar y movilizar los insumos y las partes a los lugares mas convenientes Los paises aitamente protegidos se quedarian sin empresas multi- macionales que son las que determinan el comercio imtemacional. De todas formas, existe un margen para emplear los instrumentos arancelarios y cambiarios para influir la estructura productiva Por cjemplo. una pequeña diferencia entre los aranceles de los productos finales e intermedios perminiria generar una esuructura producuva en favor de las importaciones o del alto valor agregado nacional Asi mismo. un manejo mas regulado de la tasa de cambio contriburria a promover una estructura comercial mas orientada hacia las exportaciones que hacia las actividades no transables En termunos generales. los paises todavia disponen de la discrecionalidad para una cierta regulacion de las economias que encauce las fuerzas del mercado dentro de los grandes objetur os nacionales

\section{AMFOARA}


Los paises de la region se encuentran entre la espada y la pared. De un lado estan los organismos internacionales que ios presionan para mantener las reformas estructurales de libre mercado y ahondarlas. De otro lado. estan las grandes may onas que demandan un nuevo esquema de desarrollo que le ofrezca mejores oportunidades de empleos y de salarios De hecho se plantea la solucion intermedia que, sin desconocer las realidad de la globalizacion, avance en un modelo que fovorezca a las grandes mayoras EI primer paso es revisar el modelo de sustitucion del valor agregado nacional modificando la estructura arancelaria Si adictonalmente se incrementara en pocos puntos el nivel arancelario is elevara el tipo de cambio. se conformaria una estructura productiva que favorecena las actividades transables. la agricultura y la industria Este sistema estaria liderado por actis idades que podran soportar el crecimiento sostenido de la demanda interna y externa. el ahorro y el uvance tecnologico

Muchos de los infonunios de la apertura se originan en la creencia de que la polituca comercial es un instrumento de crecimento economico s de que el desmonte arancelario pror si solo conduce a la aceleracion sostenida del crecimiento. El fracaso generalizado de tal presuncion solo ha venido a confirmar la teoria prevaleciente de tiempo atras segun la cual el crecimiento economico es un problema de ahorro o de àance tecnologico El comercio internacional es solo un suplemento que actua posituvamente en la medida que favorezca el desarrollo de los factores fundamentales

La apertura economica se convirtso precisamente en un modelo de anudesarrollo porque induce una estructura productiva que no ofrece posibilidades de absorcion tecnologica ? porque provoco una caida de la tasa de ahorro

En las circunstancias actuales de la economia, ef ahorro constituye la prncipal limitacion para elevar el crecimiento economico y ampliar las posibilidades de empleo. Esta es la diferencra sustantiva con los paises del $\mathrm{Pa}$ cifico asiatico Mientras en estos paises la tasa de ahorro asciende a $30 \%$ en America L atina no llega a $20 \%$ Si los parses de la tegion lograran tasas de ahorro de $30 \%$ o la movilizaran adecuadamentc: casi automaticamente alcanzaria tasas de crecimiento de $8 \% 0$ $y^{\circ} \circ$ durante 15 o 20 años.

La prioridad del ahorro no es nueva Otra cosa es que las formulas para clevarlo hav an fracasado sistematicamente. El ahorro es muy poco sensible a las soluciones de mercado. Tan cierto es esto que las elevadas tasas de interes que prevalecieron en la economia colombiana en las uitımas dos de- 
cadas no lograron ev itar que la tasa de ahorro se mantuviera constante y cayera drasticamente en los ultimos tres años

Los hechos se han encargado de corroborar que el ahorro solo puede aumentarse por medios forzosos que reduzcan el consumo. Tales son los casos de la tributacion progresivat las cotizaciones a la seguridad social y la conformacion de excedentes en las actividades mineras lyualmente imporlante es su orientacion. Como muchos agentes economicos estan dispucstos. durante ciertas epocas de su vida a gastar por encima del ingreso, el acceso limitado al credito factita el desplazamiento del ahorro al consumo Por eso. los esfuerzos para elevar el ahorro deben ser acompañados de regulaciones que limiten la financiacion dei consumo y garanticen la movilizacion del credito hacia las inversiones mas productivas

\section{CONCLESIONES}

El desempeño de America L.atina en las ultimas decadas no es satisfactorio. En la decada del 80 experimento una caida del ingreso per capsta. to que llevo a denominarla la epoca perdida. Ahora en los seis primeros meses de la presente decada el ingreso per capita crecio $1^{\circ}$ \% y a lo sumo crecera $1.3^{\circ}$ o en la totahdad de la decadat lo que stgmifica la perdada de las dos decadas. Al final del decento el producto interno bruto per capita sera similar al registrado a principios de los 80 . Aun mas grave. el salario real que en el actualidad es infenor al de 1980 y seguramente estas condiciones no cambiaran en lo que queda de la decada

La crisis de la deuda externa de principios de la decada del 80 y en general el mal desempento de las economas a lo largo de la decada. llevaron a buscar la solucion en las reformas estructurales de latremercado. El punto de referencia era Chile que habia adoptado esas reformas en la decada del 70 . En todos los paises se prosiguieron las reformas financiçras, se adoptaron reducciones sugnificativas de los aranceles. se libero el cambio y se iniciaron procesos de privatizacion de las empresas de strvicios publicos

Sin duda. la apertura cambiaria y comercial determino la reonentacion de la composicion productiva Todas las economas se vieron abocadas a reducciones arancelarias y revaluaciones que indujeron el desplazamiento de las actividades industrales y agricolas de alto valor agregado. Se configuro una estructura productiva ahtamente dependiente de las importaciones y con setias limitaciones en las exportaciones Aparecio un modelo en el cual la produccion nacional crece en la medida en que las imponaciones crecen mas rapi-

\section{AnFOEA}


damente. En consecuencia, los paises procedieron a ampliar la demanda para inducir mayores tasas de crecimiento y contratar creditos externos para financiar las importaciones. Las economias se vieron abocadas a crecientes deficit en cuenta corriente que no eran sostenibles. Al cabo de tres años se vicron obligadas a adoptar ajusies para revertir la tendencia cambiaria, lo que llevo a procesos recesivos que regresaron las economias a la realidad de la apertura. Sus posibilidades de expansion quedaron determinadas por ta industria y la agricultura que se vieron seriamente afectados por la apertura. durante la cual crecieron a la micad del promedio histonco. Dentro de este conlexto era evidente que la recuperacion de las tasas de crecimiento del parado solo podia lograrse medianic cuantiosos deficit en cuenta corriente yue no son sostenibies
Los mavores costos de las reformas recaycron en el sector laboral. De hecho, significan el desplazamiento del empleo por las importaciones. El efecto se compenso iniciaimente cuando las economias lograron tasas de crecimiento supenores al pasado. Sin embargo, cuando entraron en recesion y se estabilizaron en tasas de crecimiento inferiores a las del pasado, el desempleo avanzo a velocidades espectaculares. Las economias quedaron ante el dilema del desempleo, la reducción del salario. En cierta forma se demostraba que los aranceles no eran mas que un procedimiento para competir en los mercados extemos con menores sacrificios de salarios

"Que otra cosa se quera? ¿Que podia esperarse de una economia que experimenta un aumento de las importaciones que no guarda correspondencra con el crecimiento economico ni en

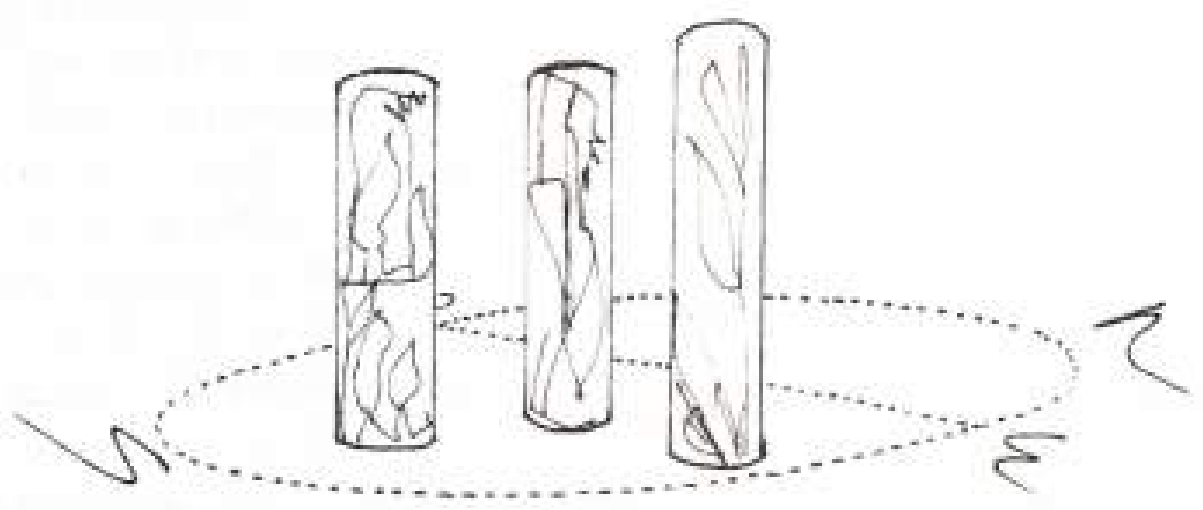


las exportaciones? A menos que la diferencia entre las importaciones ? las exportaciones se financie con expansion monetaria el resultado inevitable es un aumento de desempleo

La economia colombiana ha evolucionado dentro del mismo marco de Amenca Latina $\mathrm{Fn}$ el fondo estamos ante el mismo modelo que we ha aplicado con una uniformidad y disciplina sin precedentes En efecto la apertura comercial con revaluacion determino un desplazamiento de la industra y la agnicultura de alto valor agregado, conformo un creciente deficit en cuenta cornente y ocasiono una carda de la cuarta parte det ahorro En un principio la actividad cconomsca se acelero. a los tres años decayo $y$ en la actualidad se encuentra en plena recesion Adicionalmente, el cambio en la composicion productiva en contra del talor agregado y el menor dinamismo de la economia afectaron seriamente el sector laboral: en la agnecultura adquirio la forma de una caida del salano real

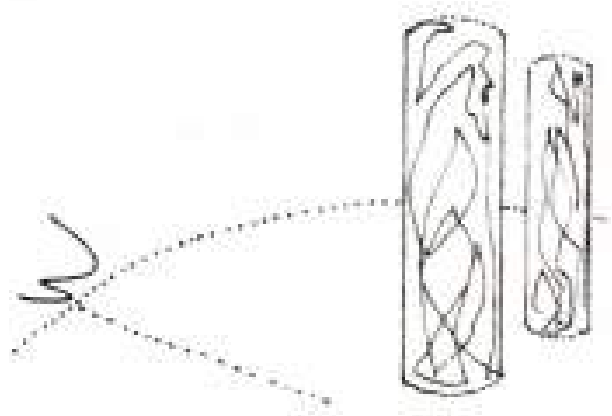

I en el sector urbano en una explosion del desempleo

La conformacion del deficit en cuenta corriente ofrecia una clara evidencla de que lis apernura no habai ocasionado los resultados previstos por la teoria la modificacion de los precios relativos determino un cambio en la estructura productiva que indujo un aumento en las importaciones muy supenor al de las exporiaciones. Como era apenas matural. aquellos que se equivocaron en la prediccion de los resultados de la apertura habrian de equivocarse mas tarde en su rectificacion. Los esfuerzos se onentaron para corregir el defict en cuenta corriente que era mas la manifestacion de la crisis Las autoridades monetarias aplicaron una severa restriccion monetaria que se manifesto en elevadas tasas de interes que. a su tumo, profundizaron la revaluacion s precipitaron la economa en recesion. Los dos efectos operaban en direccion contrana Mientras la revaluacion acentuaba las catusas estructurales del deficit, la recesion moderaba sus manifestaciones Despues de dos anos se observaba que el ajuste habia frenado la tendencla creciente del deficit en cuenta cornente, pero no habia alterado considerablemente su magnitud. En plena recesion el deficit en cuenta corriente liegaba a $50_{0}$. No es dificil imagnar que tan pronto como la economia se reactivara, el deficit tetornaria al mis-

\section{AKFGAA}


mo comportamiento anterior

Algo similar sucedio en thatera fiscal. La economia colombiana venia operando con un deficut del $3.50 \%$ del PIB en el gobiemo central y del $1.5^{\circ} \%$ del PlB en el sector publice consolidado que es el que cuenta para efectos macroeconomicos Por su parte, la recesion contribuvo a aumentair el deficit en un punto del PAB, De suerte que el deficit del sector publico consolidado llego a $2.5 \%$ del PIB Si bien era una eifra que se salia de los niveles de tolerancia, tambien es cierto que no era tan dramatica en un momento de recesion

Infortunadamente. el pais se equivoco de nuevo. No se entendio que el aumento del deficit fiscal era una manifestacion de la recesion. Encirculos influyentes se abrio camino el draenostico segun el cuat el deficit era la causa de la recesion y la revaluacion Lo cierto es que el Gobierno. influenciado por la magnitud aritmetica del deficit adopto una emergencia economica para elevar los Iributos mediante nuevos gravamenes A los dos meses de adoptar la determinacion era evidente que esta habia abortado cualquier sintoma de reacuvacion, prolongando $y$ acentuando el proceso recesivo.

El proceso se ha visto agravado por las dificultades del sistema economico a adaptarse a las reformas de hbre mercado. Cuando las autoridades monetarias reconocieron el error, intentaron revertur el comportamiento de las tasas de interes. Sus acciones para ampliar el credito no lograron bajar considerablemente las tasas de interes, en particular las tasas de interes activas. Por otra parte. las entradas de capitales ocusionaron una caida de tipo nominal de cambio que no arrastra consigo la inflacion, que tiende a ser determinada en un alto grado por la tendencra historica La cconomia entra en un proceso sistemafico de revaluación real que estimula mavores entradas de capitales y mas revaluacion real. Se configura un circulo vicioso que puede terminar en colapso

En tin, el pais se enfrenta a un grave estado recesivo que ha sido inducido por graves errores de diagnostico. No se entendio o no se quiso entender que el debilitamiento generalizado de la economia se originaba en los daños estructurales de la apertura economica, Las acciones se han orienta-

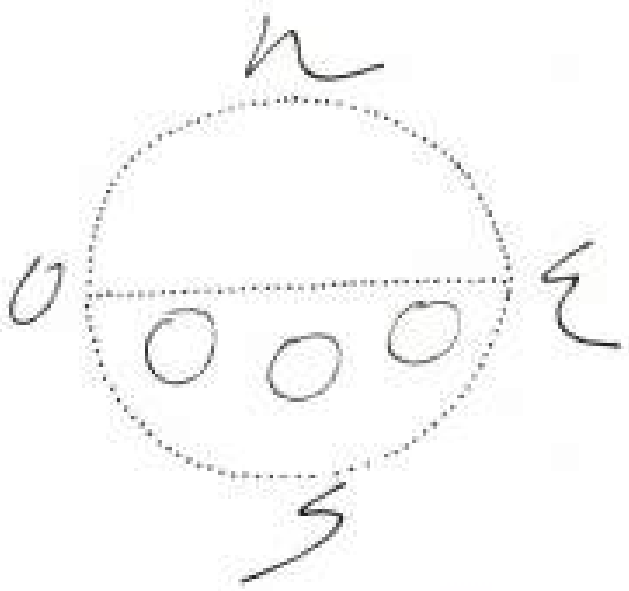


do, mas bien, a operar sobre ias manifestaciones elevando las tasas de interes e incrementando los gravamenes tributarios. Como consecuencia. las causas estructurales de la recesion se han venido acentuando y sus manifestaciones se han mongerado pasajeramente Por lo demas las deficiencias del sector tinancicto impiden los ajustes rapidos de las tasas de interes y la modalidad de banda cambiara crea sesgos en favor de la revaiuacion. Asi. el origen del deterioro de la economia esta en los daños estructurales de la apertura y en los intentos de corregir sus manifestaciones cambiarias i fiscales, y su prolonyacion y persistencia en las reformas de libre mercado que dejaron el sistema sin mecanismos de correccion. Todo esto ha resultado en una recesion que sé acentua progrestvamente y coincide con la revaluacion. tasas de interes internas superiores a las externas e inflacion simiar a la histonca. De mantenerse este marco de referencia, la economia caeria en un estado de detenoro creciente que incsitablemente termunaria en el colapso

Los resultados del modelo han modificado el discurso de sus autores y promotores en el sentido que ya no lo presentan como la panacea sino como un imperativo de la globalizacion Aur asi. la recomendacion de los organismos intemacionales es persister y ahondar en las reformas de hbre mercado hasta sus ultimas consecuencias Por otra parte, las grandes mayoras no ocultan el malestar ocasionado por el bajo dinamismo del modelo y sus sesgos en contra de los ingresos laborales. En cierta forma los parses se encuentran ante el dilema de un modelo dictado por las condiciones de la globalidad que lesiona a las grandes mayorias. La aiternativa no puede ser distinta a la de buscar un nuevo camino que dentro de los margenes de la realidad mundial contribuya a mejorar las condiciones de empleo y salarios.

El margen de maniobra para modificar el modelo economico dentro de la globalidad es limitada. No obstante. el pais esta ep condiciones de introducir cambios que le permitirian acelerar el crecimiento y corregir sus sesgos en contra de los grupos laborales. La formula consistiria en modificar la estructura arancelarta. clevar higeramente el nivel. acelerar el tipo de cambio y aumentar el ahorro por procedimicntos forzosos y canalizario hacia la inversion.

(1) 1996) SARMIENTO, Eduardo Crecimiento Economico y Apertura Economical Tercet Mundo Editores. Academia Colombuana de Cienciss Economicas. Escuela Colombiana de Ingenieria - Capituio I
(2) Comision de Racionalizacion det Gas- to y de las Finanzas Publicas informe de Avance, mareo 1996 . Veanse cuadros Iy II

\section{6 anfona}

